\title{
The Survey of Industrial Robot Vision-Guided Technology
}

\section{Fahui Liang}

Changchun automobile industry institute, Changchun, 130000, China.

1060138576@qq.com

Keywords: Industrial robot; Vision-guided; Visually capture; Dynamic grasping

\begin{abstract}
Industrial robot has become essential important equipment in advanced manufa- cturing industry. As one of the practical current robot technology research, industrial robotic vision-guided technology lays the foundation for application of the industrial robots in the smart line. Visual guidance use image information to locate the position of the target object in space, guide the direction for industrial robots to complete specific work. In this article, the development, architecture and application of the robotic vision-guided technology are described and analyzed in detail. The key technologies of industrial robot vision-guided such as visually capture, visual guidance have also been explained.
\end{abstract}

\section{Introduction}

With the improvement of automaticity and the mature development of relevant sensor technique, communications technology and artificial intelligent technique. The dependency on the fixed position and product get weaker and weaker for industry robot, which breaks traditional production pattern. Vision sensing technology are integrated into the industrial robot system so that it could make the corresponding adjustment to the varying environment in the flexible space. Furthermore, robot vision system can providing real time status information of the target objects, so it is considered the one of the most important perceptual ability. It is significant for us to apply vision system to the industrial robot to improve the intelligence level of industrial robot in the production process. Vision-guided system is based on the computer vision technology, which use image information to locate the position of the target object in space and it will guide robot to perform a certain behavior $[1,2,3,4]$.The positioning accuracy of vision system is vary important for guiding effect in this process. In China, the research of robot technology began in the late 70s. And in the 90s, China government has invested more than 20 robotic scientific research and industrialization bases. At present, the research in industrial robot has made some breakthrough, such as the robot vision system group of Tsinghua University has developed a set of CMMS multi-sensor vision system based on CAD model.

\section{The Robot Vision-Guided System}

In order to realize the supermatic industrial robot visual-guided system, a complete visual-guided system is composed by the robot system, visual system, bus system, identification system and auxiliary system. First, the images of workpiece are taken by the visual system, than visual system began to extract the feature points of image coordinate after binarization processing and work out the correcting value of workpiece coordinate system, visual system transform the results to hexadecimal data and transfer the data to robot. The data will guide the robot to establish new workpiece coordinate system. The transmission of internal analog signal in the whole system and the communication of robotic instructions are done by bus system [5]. 


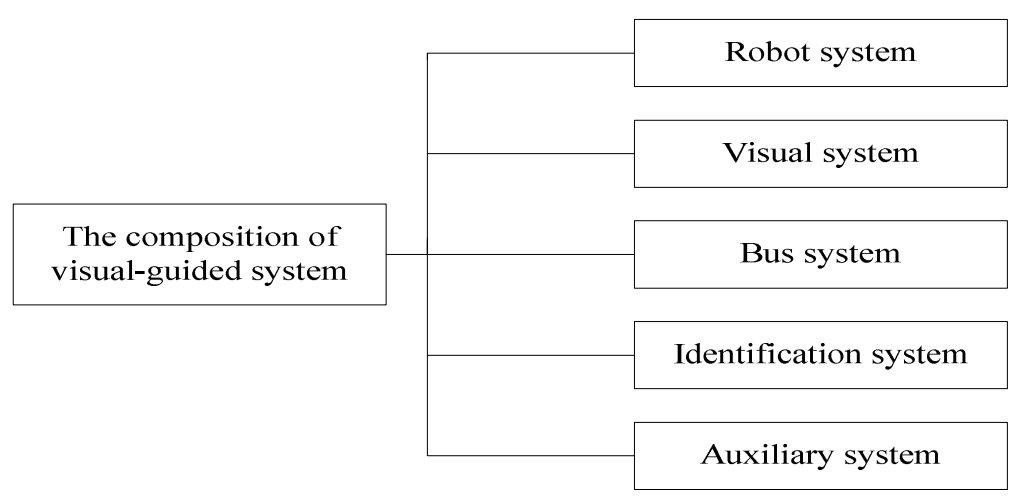

Figure 1. The composition of visual-guided system

Robot system is consist of robot body, robot control cabinet, teaching apparatus and other auxiliary equipment. This system is divided into power part and control part. Power part including six-axis motor's drive power supply, robotic PC's power supply and the controlled power supply of network part. Robot use PC technology and apply the industry standardized PC theory to control the robot, it have a complete private and common ports. Use of industry standardized PCI and ISA card slot and the corresponding function card will be very convenient to access DeviceNet, InterBus, ControlNet and other various types of industrial bus.

The execution unit of robot system is visual-guided system, and visual system is the compensation unit. The mission of the visual system is to collect workpiece images by the camera and extract image feature information. Through internal algorithm to work out the correcting value of work piece coordinate system and then transfer the data to the PLC or robot. The entire visual system including camera units, lighting system, control unit and calibration unit. Camera unit usually contains one or more cameras, shelters and installing supports. In order to obtain the spatial information, the best angle of the camera and photographed plane is 45 degrees. Control unit including industrial computer, image acquisition card, the bus communication card and display. Calibration unit including anchor slab, calibration board, ball and calibration support, etc.

The bond of controlling system is bus industry in industrial automation equipment ,the most popular applications are CAN bus, DeviceNet fieldbus, ProfiBus bus and EtherNet bus. If there are any errors for bus, it may result in the equipment's shut down and serious production's losses. There also needs to use the bus interface technology and bus coupling board to ensure that all equipment are under reliable data exchange. At the same time, in the robot control PC cabinet are reserved 5 PCI slot in order to meet the requirements of various bus, different kinds of bus technology in the implementation process just need to load the various bus protocol of the board card into the PCI slot, then further the work of network layout according to the requirements of the location wiring.

\section{The Key Technologies of Robot Vision-Guided}

Camera Calibration. According to whether or not to use target in the process of camera calibration, camera calibration study history can be divided into two stages, the one is use target, the other is don't use target. the method using target is placed a reference that is marked on a series of coordinates of feature points in the camera's field of view. Associated equipments began to establish image coordinate, camera coordinate system and world coordinate system after get the referenced image, and then extract the feature points of image coordinates. Finally, system will obtain the camera's internal and external parameters through solving these equation. The target is divided into three-dimensional and plane target, the study of three-dimensional target's calibration is more early and the precision of calibration is higher. But the production of three-dimensional target is more troublesome[6].In order to facilitate the calibration of camera, people are more likely to use planar target to realize the camera calibration on the basis of the three-dimensional target. Camera calibration of don't use target is no reference in the front of camera, it is through the specific 
movement of the camera itself to achieve the goal. In recent years, more and more researchers devote themselves to the study on the method of don't use target which have more requirements on the movement of the camera itself and working environment, how to reduce these requirements is an important aspect in the research process.

Image Segmentation. In the located workpiece image, it should separate workpieces and background region by image segmentation technology because the interested area is the workpiece itself. Image segmentation can be simply described as the process of use relevant standards to distill or segment the target workpiece and background. The target region can be regarded as the intermediary agent to analysis the follow-up images, the above work is significance for vision system. According to the manifestation of the image, it can be divided into gray-scale and color image segmentation[7]. The research of gray image segmentation is more early and a variety of image segmentation method have been proposed and have acquired good effect. In view of the different nature of gray image, the popular method of image segmentation are based on pixel's sudden changes and pixel similarity. The basic idea of based on pixel similarity is to put together the connected pixels which gray value has little difference.

Image Distortion Correction based on Calibration. Visual guide technology is to process and analysis target images to get the position of target workpiece in space, and the position of the pixels are relate to the corresponding point of the target object's surface and the position of camera. Therefore, it needs to establish corresponding relationship among image pixels, camera and space objects[8].There were distortions more or less because the camera geometric linear model is't ideal. In order to restore the ideal location of pixels in the image, we need try to reduce or even eliminate the deviation, in other words, the application of image distortion correction.

\section{Conclusion}

With the rising demands for science and technology and the increasing of labor cost, the high degree of automation and high level of production operation system which is represented by industrial robots has favored by enterprises. And it also became an important symbol of the development level of a country for its hi-tech industry. The research of robot technology bring together a series of research and development of subject, such as the visual guide technology to increase the intelligent identification capability of the robot. Visual guide technology is based on computer vision technology and identify the target object and its position in the image. This paper mainly introduces the composition of industrial robot vision-guided system and the problem about accurate positioning. The paper analyze the existing work and main technology briefly.

\section{References}

[1] Alvarez-Ramirez J, Santibañez V, Campa R. Stability of robot manipulators under saturated PID compensation[J].IEEE Transactions on Control Systems Technology, 2008, 16(6): 1333-1341.

[2] Sun D, Hu S, Shao X, et al. Global stability of a saturated nonlinear PID controller for robot manipulators[J]. IEEE Transactions on Control Systems Technology, 2009, 17(4): 892-899.

[3] Kang H J, Ro Y S. Robot manipulator modeling in Matlab-Sim Mechanics with PD control and online gravity compensation[C]. International Forum on Strategic Technology. October 13-15, 2010, Ulsan, Korea. 446-449.

[4] Su Y, Parra-Vega V. Global asymptotic saturated output feedback control of robot manipulators[C]. Proceedings of the 7th World Congress on Intelligent Control and Automation. June 25-28, 2008, Chongqing, China. 3445-3450.

[5] Cai P Z, Yuan Z S, Wei L, et al. Research on spray-paint robot position control system based on BP neural network[C]. IEEE International Conference on Robotics and Biomimetics, July 05-09, 2005, Hongkong, China. 742-746.

[6] Mc P F,Murry D W.Active Camera Calibbration for Head-eye Platform Using the VariableState-Dimension Filter. IEEE Transaction on Snalysis and Machine Intelligence, 1996, 
18(1):15-12.

[7] Xia Yong, Feng Dagan, Wang Tianjiao. Image segmentation by clustering of spatial patterns.Pattern Recognition Letters, 2007, 28(12): 1548-1555.

[8] Abutaleb A S. Automatic thresholding of gray-level pictures using two dimension entropy. Computer Vision, Graphics and Image Processing, 1989, 47(1): 22-32. 